\title{
A Simple Expression for Multivariate Lagrange Interpolation
}

Kamron Saniee, 2007

\begin{abstract}
We derive a simple formula for constructing the degree $n$ multinomial function which interpolates a set of $\left(\begin{array}{c}n+m \\ n\end{array}\right)$ points in $\mathbb{R}^{m+1}$, when the function is unique. The formula coincides with the standard Lagrange interpolation formula if the points are given in $\mathbb{R}^{2}$. We also provide examples to show how the formula is used in practice.
\end{abstract}

Key words. Lagrange interpolation, multivariate interpolation

\section{Introduction}

Interpolation, a fundamental topic in numerical analysis, is the problem of constructing a function which goes through a given set of data points. In some applications, these data points are obtained by sampling a function or process; subsequently, the values of the function can be used to construct an interpolant, which must agree with the interpolated function at the data points. The simplest kind of interpolation, in which most development has been made, is interpolation by means of univariate polynomials. Multiple formulae for polynomial interpolation have been given, notably those of Newton and Lagrange [1].

Multivariate interpolation has applications in computer graphics, numerical quadrature, cubature, and numerical solutions to differential equations $[2,3]$. The purpose of this paper is to give an explicit multivariate analogue of Lagrange's formula, under conditions which we will specify.

\section{Polynomial Interpolation}

Lagrange gave the following interpolation polynomial $p(X)$ of degree $n$ given $n+1$ points $\left(x_{i}, y_{i}\right)_{i=1}^{n+1} \in$ $\mathbb{R}^{2}$ where $x_{i} \neq x_{j}$ :

$$
y=p(X)=\sum_{i=1}^{n+1} y_{i} \ell_{i}(X)
$$

where the $\ell_{i}(X)$ are the Lagrange Basis Polynomials, defined by

$$
\ell_{i}(X)=\prod_{j=1}^{n+1} \frac{X-x_{j}}{x_{i}-x_{j}} .
$$

An interesting feature of this formula, and the feature we aim to preserve in generalizing it, is that when we substitute $x_{i}$ for $X, \ell_{i}\left(x_{i}\right)=1$ and $\ell_{j}\left(x_{i}\right)=0(j \neq i)$, giving $y=y_{i}$. The advantage of

\footnotetext{
${ }^{*}$ New Providence High School, New Providence, NJ 07974
} 
this form of the interpolation polynomial is that we can write down the interpolant immediately, without computing the coefficients in the function.

On the other hand, Newton's interpolation polynomial is of the form

$$
p(X)=\sum_{i=1}^{n+1} a_{i} n_{i}(X)
$$

where $n_{1}(X) \equiv 1, n_{i}(X)=\prod_{j=1}^{i-1}\left(X-x_{j}\right)_{i=2}^{n+1}$ and the coefficients $a_{i} \quad(1 \leq i \leq n+1)$ are defined as $a_{i}=\left[y_{1}, \ldots, y_{i}\right]$, where $\left[y_{1}, \ldots, y_{i}\right]$ are divided differences, defined recursively by $\left[y_{j}\right]=y_{j}$, and $\left[y_{j}, \ldots, y_{k}\right]=\frac{\left[y_{j+1}, \ldots, y_{k}\right]-\left[y_{j}, \ldots, y_{k-1}\right]}{y_{k}-y_{j}}$.

Unlike Lagrange's formula, Newton's does not give an explicit form for the interpolant until after the divided differences are computed. On the other hand, this form conveniently accommodates changes in the data set, for the basis polynomials do not need to be completely recalculated.

The uniqueness of the interpolation polynomial follows from the Fundamental Theorem of Algebra (if there were another polynomial function $g(X)$ of degree not exceeding $n$ coinciding with $p(X)$ at $\left(x_{i}, y_{i}\right)_{i=1}^{n+1}$, then $p(X)-g(X)$ would be a polynomial function of degree not exceeding $n$ with $n+1$ roots, and must thus be zero).

\section{The Multivariate Case}

Let $f=f\left(X_{1}, \ldots, X_{m}\right)$ be an $m$-variable multinomial function of degree $n$. Since there are $\left(\begin{array}{c}n+m \\ n\end{array}\right)=\rho$ terms in $f$ (see appendix), it is a necessary condition that we have $\rho$ distinct points $\left(x_{1, i}, \ldots, x_{m, i}, f_{i}\right) \in \mathbb{R}^{m+1}, 1 \leq i \leq \rho, f_{i}=f\left(x_{1, i}, \ldots, x_{m, i}\right)$, for $f$ to be uniquely defined. In other words,

$$
f\left(X_{1}, \ldots, X_{m}\right)=\sum_{\mathbf{e}_{i} \cdot \mathbf{1} \leq n} \alpha_{\mathbf{e}_{i}} \mathbf{X}^{\mathbf{e}_{i}}
$$

where the $\alpha_{\mathbf{e}_{i}}$ are the coefficients in $f, \mathbf{X}=\left(X_{1}, \ldots, X_{m}\right)$ is the $m$-tuple of independent variables of $f, \mathbf{e}_{i}=\left(e_{1 i}, \ldots, e_{m i}\right)$ is an exponent vector with nonnegative integer entries consisting of an ordered partition of an integer between 0 and $n$ inclusive, $\mathbf{e}_{i} \cdot \mathbf{1}:=\sum_{j=1}^{m} e_{j i}$ is the usual vector dot product, and $\mathbf{X}^{\mathbf{e}_{i}}:=\prod_{j=1}^{m} X_{j}^{e_{j i}}$. Following Lagrange, we wish to write $f$ in the form $\sum_{i=1}^{\rho} f_{i} \ell_{i}(\mathbf{X})$, where $\ell_{i}(\mathbf{X})$ is a multinomial function in the independent variables $X_{1}, \ldots, X_{m}$ with the property that when $\mathbf{X}$ is equal to the $i^{\text {th }}$ data value, or $\mathbf{X}=\mathbf{x}_{i}\left(\left(X_{1}, \ldots, X_{m}\right)=\left(x_{1, i}, \ldots, x_{m, i}\right)\right)$, then $\ell_{i}\left(\mathbf{x}_{i}\right)=1$ and $\ell_{j}\left(\mathbf{x}_{i}\right)=0(j \neq i)$. To do this, consider the system of linear equations $f_{i}=\sum_{\mathbf{e}_{j} \cdot \mathbf{1} \leq n} \alpha_{\mathbf{e}_{j}} \mathbf{x}_{i}^{\mathbf{e}_{j}}$, where 
$1 \leq i \leq \rho$. From this system construct the sample matrix $M=\left[\mathbf{x}_{i}^{\mathbf{e}_{j}}\right]$ :

$$
M=\left(\begin{array}{ccc}
\mathbf{x}_{1}^{\mathbf{e}_{1}} & \ldots & \mathbf{x}_{1}^{\mathbf{e}_{\rho}} \\
\vdots & & \vdots \\
\mathbf{x}_{i}^{\mathbf{e}_{1}} & \ldots & \mathbf{x}_{i}^{\mathbf{e}_{\rho}} \\
\vdots & & \vdots \\
\mathbf{x}_{\rho}^{\mathbf{e}_{1}} & \ldots & \mathbf{x}_{\rho}^{\mathbf{e}_{\rho}}
\end{array}\right)
$$

We assume $\operatorname{det}(M) \neq 0$. Although we could solve for the coefficients $\alpha_{i}$ in $f$ by inverting $M$ (which we know to be square because the number of equations in the above linear system is equal to the number of coefficients), that is not our goal; the utility of Lagrange interpolation is that we can in fact determine $f$ without explicitly solving for its coefficients.

Remark. If $M$ is singular, then the coefficients of $f$ are not uniquely determined, in which case $f$ is clearly not unique. Therefore, $f$ is unique if and only if its sample matrix is nonsingular. On the other hand, characterizing the geometric configuration of the $\rho$ points so that $\operatorname{det}(M)=0$ appears to be an intricate problem [2].

Let $\Delta=\operatorname{det}(M)$. Now make the substitutions $\mathbf{x}_{j}=\mathbf{X}$ in $M$; this gives the following matrix $M_{j}(\mathbf{X})$ :

$$
M_{j}(\mathbf{X})=\left(\begin{array}{ccc}
\mathbf{x}_{1}^{\mathbf{e}_{1}} & \ldots & \mathbf{x}_{1}^{\mathbf{e}_{\rho}} \\
\vdots & & \vdots \\
\mathbf{X}^{\mathbf{e}_{1}} & \ldots & \mathbf{X}^{\mathbf{e}_{\rho}} \\
\vdots & & \vdots \\
\mathbf{x}_{\rho}^{\mathbf{e}_{1}} & \ldots & \mathbf{x}_{\rho}^{\mathbf{e}_{\rho}}
\end{array}\right) \leftarrow j^{t h} \text { row }
$$

Let $\Delta_{j}(\mathbf{X})=\operatorname{det}\left(M_{j}(\mathbf{X})\right)$. Next, make the substitutions $\mathbf{X}=\mathbf{x}_{i}$ in $M_{j}(\mathbf{X})(i \neq j)$; this gives the following matrix $\left(M_{j}\right)_{i}$ :

$$
\left(M_{j}\right)_{i}=\left(\begin{array}{ccc}
\mathbf{x}_{1}^{\mathbf{e}_{1}} & \ldots & \mathbf{x}_{1}^{\mathbf{e}_{\rho}} \\
\vdots & & \vdots \\
\mathbf{x}_{i}^{\mathbf{e}_{1}} & \ldots & \mathbf{x}_{i}^{\mathbf{e}_{\rho}} \\
\vdots & & \vdots \\
\mathbf{x}_{i}^{\mathbf{e}_{1}} & \ldots & \mathbf{x}_{i}^{\mathbf{e}_{\rho}} \\
\vdots & & \vdots \\
\mathbf{x}_{\rho}^{\mathbf{e}_{1}} & \ldots & \mathbf{x}_{\rho}^{\mathbf{e}_{\rho}}
\end{array}\right) \leftarrow i^{\text {th }} \text { row }
$$

Note that the $i^{\text {th }}$ row appears twice in $\left(M_{j}\right)_{i}$. That means $\operatorname{det}\left(\left(M_{j}\right)_{i}\right)=0$. In other words, when $\mathbf{X}=\mathbf{x}_{i}$ then $\Delta_{j}\left(\mathbf{x}_{i}\right)=0(i \neq j)$. By construction, moreover, $\mathbf{X}=\mathbf{x}_{i} \Rightarrow \Delta_{i}(\mathbf{X})=\Delta$. Hence,

$$
\ell_{i}(\mathbf{X})=\frac{\Delta_{i}(\mathbf{X})}{\Delta}
$$

and therefore

$$
f=\sum_{i=1}^{\rho} f_{i} \frac{\Delta_{i}(\mathbf{X})}{\Delta} .
$$


Example 1. Suppose we are given three data points $(0,0,1),(0,1,2)$, and $(1,1,3)$ that lie on $z=f(x, y)(m=2)$. These points define uniquely a linear function of two variables, so $z_{i}=$ $\alpha_{1} x_{i}+\alpha_{2} y_{i}+\alpha_{3}, 1 \leq i \leq 3$, for some coefficients $\alpha_{1}, \alpha_{2}, \alpha_{3}\left(n=1, \rho=\left(\begin{array}{c}2+1 \\ 1\end{array}\right)=3\right)$. Hence the coefficients must satisfy

$$
\begin{array}{lllll}
1 & = & & & \\
\alpha_{3} \\
2 & = & & & \alpha_{2}+\alpha_{3} \\
3 & = & \alpha_{1}+\alpha_{2}+\alpha_{3}
\end{array}
$$

From (4) it follows that

$$
M=\left(\begin{array}{lll}
0 & 0 & 1 \\
0 & 1 & 1 \\
1 & 1 & 1
\end{array}\right)
$$

and from (5)

$$
M_{1}=\left(\begin{array}{lll}
x & y & 1 \\
0 & 1 & 1 \\
1 & 1 & 1
\end{array}\right) \quad M_{2}=\left(\begin{array}{lll}
0 & 0 & 1 \\
x & y & 1 \\
1 & 1 & 1
\end{array}\right) \quad M_{3}=\left(\begin{array}{lll}
0 & 0 & 1 \\
0 & 1 & 1 \\
x & y & 1
\end{array}\right)
$$

where $\Delta=\operatorname{det}(M)=-1, \quad \Delta_{1}=\operatorname{det}\left(M_{1}\right)=y-1, \quad \Delta_{2}=\operatorname{det}\left(M_{2}\right)=x-y, \quad \Delta_{3}=\operatorname{det}\left(M_{3}\right)=-x$. By (7), we get $z=z_{1} \frac{\Delta_{1}}{\Delta}+z_{2} \frac{\Delta_{2}}{\Delta}+z_{3} \frac{\Delta_{3}}{\Delta}=(1-y)+2(y-x)+3 x=x+y+1$.

Example 2. Suppose we are given points $(0,1,-7),(2,1,3),(1,3,-10),(-2,-1,11),(-3,2,1),(-1,2,-$ 11) that lie on $z=f(x, y)(m=2)$. These points define uniquely a degree two function of two variables, so $z_{i}=\alpha_{1} x_{i}^{2}+\alpha_{2} x_{i} y_{i}+\alpha_{3} y_{i}^{2}+\alpha_{4} x_{i}+\alpha_{5} y_{i}+\alpha_{6}$, for coefficients $\alpha_{1}, \ldots, \alpha_{6}$, and $1 \leq i \leq 6$ $\left(n=2, \rho=\left(\begin{array}{c}2+2 \\ 2\end{array}\right)=6\right)$. Thus

$$
\begin{aligned}
& -7=\alpha_{3}+\alpha_{5}+\alpha_{6} \\
& 3=4 \alpha_{1}+2 \alpha_{2}+\alpha_{3}+2 \alpha_{4}+\alpha_{5}+\alpha_{6} \\
& -10=\alpha_{1}+3 \alpha_{2}+9 \alpha_{3}+\alpha_{4}+3 \alpha_{5}+\alpha_{6} \\
& 11=4 \alpha_{1}+2 \alpha_{2}+\alpha_{3}-2 \alpha_{4}-\alpha_{5}+\alpha_{6} \\
& 1=9 \alpha_{1}-6 \alpha_{2}+4 \alpha_{3}-3 \alpha_{4}+2 \alpha_{5}+\alpha_{6} \\
& -11=\alpha_{1}-2 \alpha_{2}+4 \alpha_{3}-\alpha_{4}+2 \alpha_{5}+\alpha_{6}
\end{aligned}
$$

from which

$$
\begin{gathered}
M=\left(\begin{array}{cccccc}
0 & 0 & 1 & 0 & 1 & 1 \\
4 & 2 & 1 & 2 & 1 & 1 \\
1 & 3 & 9 & 1 & 3 & 1 \\
4 & 2 & 1 & -2 & -1 & 1 \\
9 & -6 & 4 & -3 & 2 & 1 \\
1 & -2 & 4 & -1 & 2 & 1
\end{array}\right) \\
M_{1}=\left(\begin{array}{cccccc}
x^{2} & x y & y^{2} & x & y & 1 \\
4 & 2 & 1 & 2 & 1 & 1 \\
1 & 3 & 9 & 1 & 3 & 1 \\
4 & 2 & 1 & -2 & -1 & 1 \\
9 & -6 & 4 & -3 & 2 & 1 \\
1 & -2 & 4 & -1 & 2 & 1
\end{array}\right) \quad M_{2}=\left(\begin{array}{cccccc}
0 & 0 & 1 & 0 & 1 & 1 \\
x^{2} & x y & y^{2} & x & y & 1 \\
1 & 3 & 9 & 1 & 3 & 1 \\
4 & 2 & 1 & -2 & -1 & 1 \\
9 & -6 & 4 & -3 & 2 & 1 \\
1 & -2 & 4 & -1 & 2 & 1
\end{array}\right)
\end{gathered}
$$




$$
\begin{gathered}
M_{3}=\left(\begin{array}{cccccc}
0 & 0 & 1 & 0 & 1 & 1 \\
4 & 2 & 1 & 2 & 1 & 1 \\
x^{2} & x y & y^{2} & x & y & 1 \\
4 & 2 & 1 & -2 & -1 & 1 \\
9 & -6 & 4 & -3 & 2 & 1 \\
1 & -2 & 4 & -1 & 2 & 1
\end{array}\right) \quad M_{4}=\left(\begin{array}{cccccc}
0 & 0 & 1 & 0 & 1 & 1 \\
4 & 2 & 1 & 2 & 1 & 1 \\
1 & 3 & 9 & 1 & 3 & 1 \\
x^{2} & x y & y^{2} & x & y & 1 \\
9 & -6 & 4 & -3 & 2 & 1 \\
1 & -2 & 4 & -1 & 2 & 1
\end{array}\right) \\
M_{5}=\left(\begin{array}{cccccc}
0 & 0 & 1 & 0 & 1 & 1 \\
4 & 2 & 1 & 2 & 1 & 1 \\
1 & 3 & 9 & 1 & 3 & 1 \\
4 & 2 & 1 & -2 & -1 & 1 \\
x^{2} & x y & y^{2} & x & y & 1 \\
1 & -2 & 4 & -1 & 2 & 1
\end{array}\right) \quad M_{6}=\left(\begin{array}{cccccc}
0 & 0 & 1 & 0 & 1 & 1 \\
4 & 2 & 1 & 2 & 1 & 1 \\
1 & 3 & 9 & 1 & 3 & 1 \\
4 & 2 & 1 & -2 & -1 & 1 \\
9 & -6 & 4 & -3 & 2 & 1 \\
x^{2} & x y & y^{2} & x & y & 1
\end{array}\right)
\end{gathered}
$$

and

$$
\begin{aligned}
\Delta & =200 \\
\Delta_{1} & =60 x^{2}+460 x y-460 y^{2}-680 x+1360 y-700 \\
\Delta_{2} & =-12 x^{2}-172 x y+152 y^{2}+296 x-492 y+340 \\
\Delta_{3} & =24 x^{2}+144 x y-104 y^{2}-192 x+384 y-280 \\
\Delta_{4} & =-8 x^{2}-48 x y+68 y^{2}+64 x-228 y+160 \\
\Delta_{5} & =40 x^{2}+140 x y-140 y^{2}-220 x+440 y-300 \\
\Delta_{6} & =-104 x^{2}-524 x y+484 y^{2}+732 x-1464 y+980 .
\end{aligned}
$$

Now

$$
\begin{aligned}
z & =-7 \frac{\Delta_{1}}{\Delta}+3 \frac{\Delta_{2}}{\Delta}-10 \frac{\Delta_{3}}{\Delta}+11 \frac{\Delta_{4}}{\Delta}+\frac{\Delta_{5}}{\Delta}-11 \frac{\Delta_{6}}{\Delta} \\
& =2 x^{2}+x y-4 y-3 .
\end{aligned}
$$

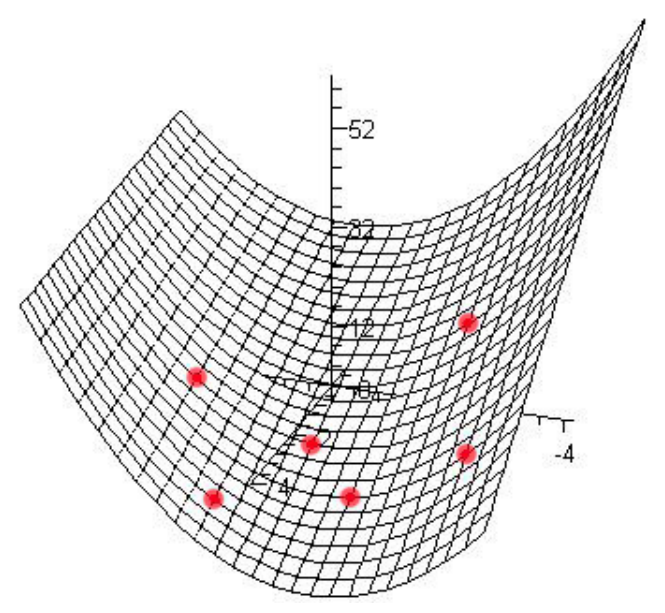


Remark. In the approach presented above, there is nothing particular about the multinomial basis. In other words, the formula (7) applies to multivariate functions of arbitrary basis, assuming the given set of data points defines the function uniquely.

Example. Suppose we are given points $(0,0,0,10),(1,1,0, e+9),(0,1,1,3 e+7),\left(2,0,1,2 e^{2}+8\right)$ which lie on a function $f\left(x_{1}, x_{2}, x_{3}\right)=\alpha_{1} e^{x_{1} x_{2}}+\alpha_{2} e^{x_{1} x_{3}}+\alpha_{3} e^{x_{2} x_{3}}+\alpha_{4}$, where the $\alpha_{i}(1 \leq i \leq 4)$ are coefficients. We have

$$
\begin{array}{ccccccccc}
10 & = & \alpha_{1} & + & \alpha_{2} & + & \alpha_{3} & + & \alpha_{4} \\
e+9 & = & \alpha_{1} e & + & \alpha_{2} & + & \alpha_{3} & + & \alpha_{4} \\
3 e+7 & = & \alpha_{1} & + & \alpha_{2} & + & \alpha_{3} e & + & \alpha_{4} \\
2 e^{2}+8 & = & \alpha_{1} & + & \alpha_{2} e^{2} & + & \alpha_{3} & + & \alpha_{4}
\end{array}
$$

and

$$
\begin{aligned}
& M=\left(\begin{array}{cccc}
1 & 1 & 1 & 1 \\
e & 1 & 1 & 1 \\
1 & 1 & e & 1 \\
1 & e^{2} & 1 & 1
\end{array}\right) \\
& M_{1}=\left(\begin{array}{cccc}
e^{x_{1} x_{2}} & e^{x_{1} x_{3}} & e^{x_{2} x_{3}} & 1 \\
e & 1 & 1 & 1 \\
1 & 1 & e & 1 \\
1 & e^{2} & 1 & 1
\end{array}\right) \quad M_{2}=\left(\begin{array}{cccc}
1 & 1 & 1 & 1 \\
e^{x_{1} x_{2}} & e^{x_{1} x_{3}} & e^{x_{2} x_{3}} & 1 \\
1 & 1 & e & 1 \\
1 & e^{2} & 1 & 1
\end{array}\right) \\
& M_{3}=\left(\begin{array}{cccc}
1 & 1 & 1 & 1 \\
e & 1 & 1 & 1 \\
e^{x_{1} x_{2}} & e^{x_{1} x_{3}} & e^{x_{2} x_{3}} & 1 \\
1 & e^{2} & 1 & 1
\end{array}\right) \quad M_{4}=\left(\begin{array}{cccc}
1 & 1 & 1 & 1 \\
e & 1 & 1 & 1 \\
1 & 1 & e & 1 \\
e^{x_{1} x_{2}} & e^{x_{1} x_{3}} & e^{x_{2} x_{3}} & 1
\end{array}\right)
\end{aligned}
$$

from which

$$
\begin{aligned}
\Delta & =(e+1)(e-1)^{3} \\
\Delta_{1} & =(e-1)^{2}\left((e+1)^{2}-(e+1)\left(e^{x_{1} x_{2}}+e^{x_{2} x_{3}}\right)-e^{x_{1} x_{3}}\right) \\
\Delta_{2} & =(e+1)(e-1)^{2}\left(e^{x_{1} x_{2}}-1\right) \\
\Delta_{3} & =(e+1)(e-1)^{2}\left(e^{x_{2} x_{3}}-1\right) \\
\Delta_{4} & =(e-1)^{2}\left(e^{x_{1} x_{3}}-1\right) .
\end{aligned}
$$

Finally,

$$
\begin{aligned}
f & =10 \frac{\Delta_{1}}{\Delta}+(e+9) \frac{\Delta_{2}}{\Delta}+(3 e+7) \frac{\Delta_{3}}{\Delta}+\left(2 e^{2}+8\right) \frac{\Delta_{4}}{\Delta} \\
& =e^{x_{1} x_{2}}+2 e^{x_{1} x_{3}}+3 e^{x_{2} x_{3}}+4 .
\end{aligned}
$$

Conclusion. We derived an explicit multivariate analogue of Lagrange's interpolation polynomial. Specifically, we showed how to interpolate an $m$-variable multinomial function of degree $n$ given 
$\left(\begin{array}{c}n+m \\ n\end{array}\right)$ distinct points. We also gave a necessary and sufficient condition for uniqueness of the interpolant, namely that the determinant of the corresponding sample matrix be nonzero. Finally, we provided numerical examples to illustrate the use of the derived expression. As mentioned in the Remark in Section 3, the geometric configurations of points that result in a singular sample matrix deserve further investigation.

Acknowledgement. The author wishes to thank the anonymous referees for valuable suggestions. In particular, the approach presented in the Appendix considerably simplified the original proof. 


\section{Appendix}

The general term in a $m$-variable multinomial function of degree $n$ is $\prod_{i=1}^{m} X_{i}^{e_{i}}$, where the $X_{i}$ are independent variables and the $e_{i}$ nonnegative integer exponents. Because $\sum_{i=1}^{m} e_{i} \leq n$, the problem of determining the the number of points needed to uniquely define $f$ is equivalent to counting the total number of ordered partitions of integers between 0 and $n$ inclusive into $m$ nonnegative parts. We represent such a partition as an arrangement of $m$ bars and $n$ stars; for example, let $m=4$ and $n=8$. The arrangement

$$
* *|| * * *|* *| *
$$

represents the partition $(2,0,3,2)$ of 7 into 4 parts, where the number of stars preceding the first bar is the first part, the number of stars between the first and second bars is the second part,..., and in general, the number of stars $k$ succeeding the last bar indicates a partition of $n-k$. Indeed, any such arrangement is a string of $n+m$ terms, with $m$ of them bars; we need only choose which $m$ terms are bars. Hence, the desired number of partitions is equal to $\left(\begin{array}{c}n+m \\ m\end{array}\right)=\left(\begin{array}{c}n+m \\ n\end{array}\right)$, as required. 


\section{References}

[1] M. Gasca and T. Sauer. Polynomial interpolation in several variables. Advances in Computational Mathematics, 12(4):377-410, 2000.

[2] P.J. Olver. On multivariate interpolation. Studies in Applied Mathematics, 116(4):201-240, 2006.

[3] J.F. Steffensen. Interpolation. Dover Publications, Inc., New York, second edition, 2006. 